\title{
A Cached Registration Scheme for IP Multimedia Subsystem (IMS)
}

\author{
Lava Al-Doski ${ }^{1}$ and Seshadri Mohan ${ }^{2}$ \\ ${ }^{1}$ NIKSUN,inc 100 Nassau park Blvd. Princeton, NJ, 08540, USA \\ ${ }^{2}$ Systems Engineering Department, EIT 519 University of Arkansas at Little Rock \\ 2801 S University Avenue, Little Rock, AR 72204, USA \\ lava.aldoski@gmail.com,sxmohan@ualr.edu
}

Received 17 August 2014; Accepted 11 September 2014

Publication 7 October 2014

\begin{abstract}
IP Multimedia Subsystem (IMS), an architectural framework for delivering multimedia services, was standardized by $3 \mathrm{GPP} / 3 \mathrm{GPP} 2$. It is integrated with $4 \mathrm{G}$ and will most likely be with $5 \mathrm{G}$ and beyond, so as to enable wireless carriers to provide rich multimedia services, such as IPTV, chat, push to talk, and video conference. To use these services, users need to perform registration procedure with IMS, which will provide user information to the system. Registration is also performed when users move from one network to another. Due to the complexity of IMS and the increasing demands for these services, a key challenge IMS faces is to provide QoS to meet user requirements. One of the key performance factors is delay encountered in registration and service establishment. Also, users' mobility impacts the rate of registration and consequently the signaling generated that must be handled by the system. This work proposes and analyzes a cached registration scheme to reduce the delay associated with registration. The work also examines the effects of different mobility models on the application layer, in particular IMS. The work studies the impact of user movement patterns on the system and examines the impact of the proposed cached registration scheme on these patterns.
\end{abstract}

Keywords: IP Multimedia Subsystem, QoS, Mobility Models, IMS cached registration scheme.

Journal of Cyber Security, Vol. 3, 317-338.

doi: $10.13052 /$ jcsm2245-1439.334

(c) 2014 River Publishers. All rights reserved. 


\section{Introduction}

IP Multimedia System has been standardized for the purpose of providing a services infrastructure for $3 \mathrm{G}, 4 \mathrm{G}$ and future wireless networks yet to evolve. IMS was created by $3 \mathrm{GPP} / 3 \mathrm{GPP} 2$ to be part of the transition to the Next Generation Network (NGN). IMS offers a wide range of services including IPTV, Push-to-Talk, Web Services, and presence. IMS infrastructure consists of a collection of servers, each serving a dedicated task. These servers deploy a variety of protocols to set up and manage services. In order to avail of IMS services, users are required to register first with the system. The registration procedure also occurs whenever the users move from one network to another or from one IMS administrative domain to another within the same network. Due to the complexity of IMS signaling, delays are expected due to queuing and processing of signaling messages. Service providers face many challenges in guaranteeing QoS requested by the user. Several issues arise that impact the delivery of required QoS. One of these issues is the impact of users' mobility patterns. These patterns determine the handover rate that affects the system. As users move from one network to another, they may be handed over from one IMS services domain to another, which will initiate registration with the new IMS. This paper proposes and analyzes a scheme that can minimize the overall delay experienced by users due to the registration process and thereby facilitate the delivery of QoS required by the users.

Literature review identified several papers that analyze the delays encountered within the IMS infrastructure. In [1], the authors investigate end-to-end SIP delay in WiMax- UMTS, WiMax - WiMax, UMTS-UMTS network. The results demonstrate that the processing delay is a key contributor [2] the author implemented a different kind of analysis. While the IMS worked within CDMA2000 environment, the authors performed end-to-end session setup delay analysis by breaking it down along the various layers and calculating the delay in each layer taking into consideration the delay property of each layer. These analyses are crucial for real time services and interactive services. In [3], the authors have suggested caching the routing data in I-CSCF. The study indicated that by utilizing the I-CSCF cache, the average incoming call setup time can be reduced.

In the literature review analyses considering user mobility have assumed mainly two mobility models (random walk, fluid flow) as a generic model to study the effect of delay on a system. These models do not represent the whole picture of user mobility patterns and therefore fail in offering the best representation of the handover delay effects. Very few studies have 
been undertaken to explore the effects of different mobility models on the application layer. In this study, we propose a cached registration scheme for IMS users with mobility patterns associated with high handover rates and then analyze the impact of different mobility models on handover rate (registration rate) encountered by IMS. Ref. [17] provides an excellent analysis of mobility management from the perspective of system optimization.

The paper is organized as follows. Section II provides a brief overview of IMS architecture. Section III proposes a cached registration scheme, and considers several mobility models. Section IV describes the approach to modeling, simulation setup and an analysis. Section V discusses results and Section VI provides some concluding remarks.

\section{IP Multimedia Subsystem Architecture}

IMS consists of a set of core servers called Call Session Control Function servers, specifically, Proxy, Interrogating, and Serving Call Session Control Functions (P, I, S-CSCF). Each of these servers has its own functionality within the system. IMS also has its own data base to keep the users' authentication tokens and profile information. The focus of this paper is on the signaling load encountered by these servers and a scheme that serves to reduce the load. The P-CSCF serves as the first point of contact with the user and it is usually located in the visitor network. The I-CSCF mainly performs routing of the IMS messages. S-CSCF may be the considered the brain of IMS and the key decision maker. S-CSCF performs user authentication and registration, and oversees establishment, monitoring and tearing down of services. Both I-, and $\mathrm{S}-\mathrm{CSCF}$ are located in the home network. IMS is connected to an assembly of servers denoted by application servers. These servers are responsible for providing the resource for IMS core servers to establish a service. Each server(s) is dedicated to a specific service. This collection can be part of IMS internal system or may belong to a $3^{\text {rd }}$ party as shown in Figure 1 [4].

As shown in Figure 2, IMS users need to perform registration procedure with the system before establishing any service. This process establishes a connection with the user, loads user profile, performs authentication, allocates resources, and establishes security agreements [5]. The registration process proceeds as follows:

1. The user sends REGISTER to P-CSCF located in the visitor network. The proxy performs Domain Name System (DNS) query for the I-CSCF URI, and then forwards the REGISTER message. 


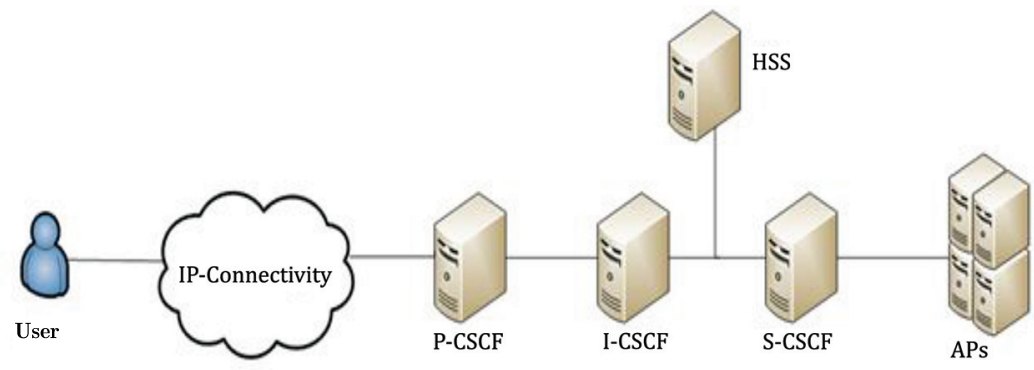

Figure 1 IMS architecture

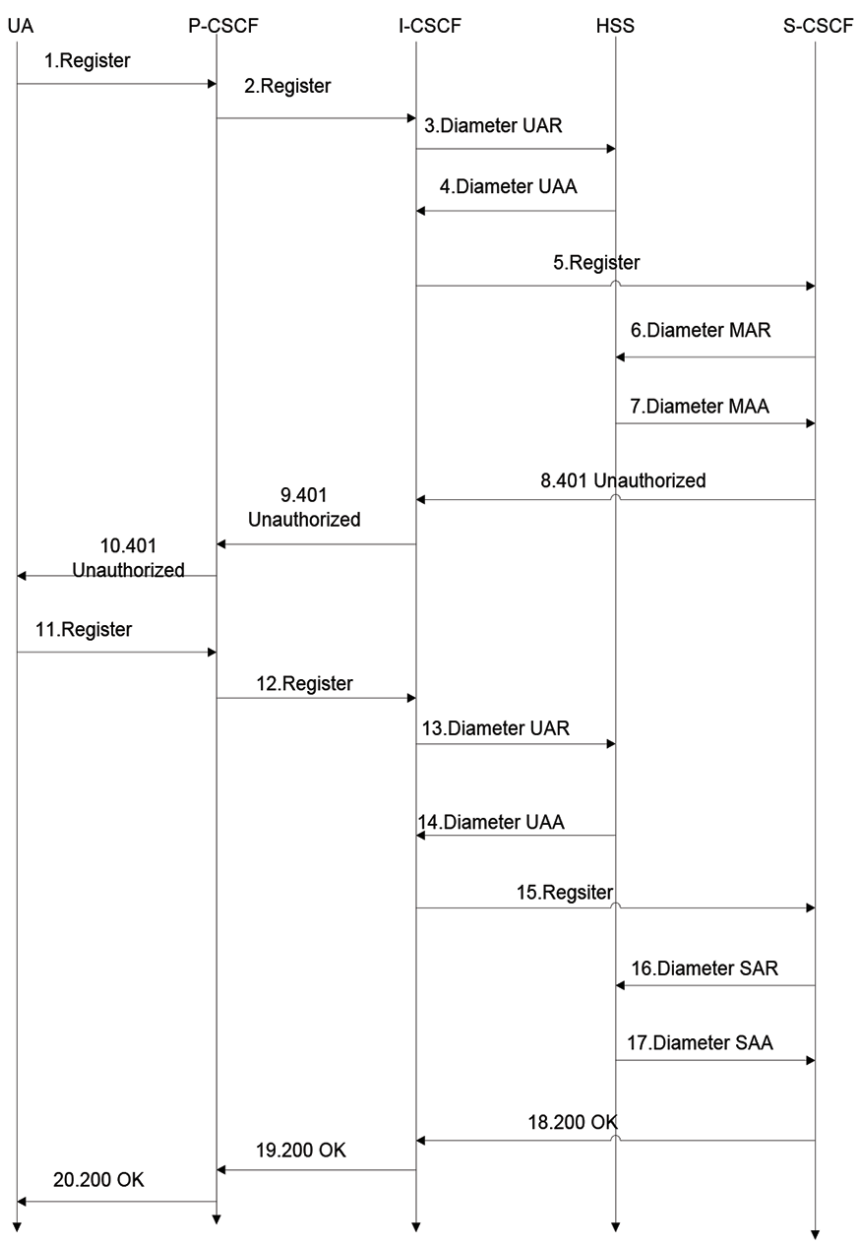

Figure 2 IMS registration procedure 
The proxy inserts its address in the forwarded message to make sure that it receives back SIP response methods to its own address.

2. The I-CSCF serves as a router and a load balancer. The I-CSCF will examine the REGISTER message and extract the user public and private identities and network ID and then sends the information via Diameter protocol User-Authentication Request (UAR) to the HSS [6].

3. On receiving the message, the HSS processes the information and verifies the roaming agreement (in the case of receiving a visitor network ID).

4. The HSS authenticates the user and assigns a S-CSCF to the user. It then sends the response in a Diameter User-Authentication Answer (UAA).

5. The I-CSCF receives the UAA and extracts the S-CSCF address and then forwards SIP REGISTER to it.

6. The S-CSCF receives the REGISTER message and sends Multimedia Authorization Answer (MAA) to the HSS. The HSS stores the S-CSCF URI.

7. The HSS sends MAA back to the S-CSCF which contains authentication information of the user.

8, 9, 10- As part of the security system, IMS users are required to accept a challenge. Basically, IMS proposes a challenge for the user to respond, a way of protecting the system from flooding with fake users' request. So the user in this stage is not authorized.

11. The user sends a registration request again with the challenge response, which will be passed back through the same route.

12, 13- In the second attempt, the I-CSCF sends registration information to the HSS in the form of UAR.

14. The HSS sends back UAA which includes the address of the S-CSCF allocated to the user.

15. The I-CSCF sends the REGISTER to the S-CSCF with the user information.

16. The S-CSCF receives the user information and sends SAR to notify the HSS that the user is registered and authenticated.

17. The HSS receives SAR and sends the user profile, user public identity and the initial filter criteria to indicate the application server URI that would be involved with the user.

$18,19,20$ - the S-CSCF sends 200 OK to indicate the registration procedure was successful. 


\section{Proposed Cached Registration Scheme}

The volume of signaling associated with registration could reach substantial scales especially in crowded areas with hybrid networks triggering frequent handovers. These registration signaling can adversely impact the QoS.

When a service is interrupted or a handover is about to occur, the $\mathrm{P}-\mathrm{CSCF}$ receives a graceful or ungraceful disconnect of service. This disconnect request is sent to the P-CSCF. The P-CSCF will inform the S-CSCF of the discontinuity of service through the I-CSCF. Subsequently, if the user's terminal performs a handover the user will send a registration request. The user might also send a re-establishment of a session if the session was interrupted during handover. Figure 3 depicts the flow diagram of the registration procedure where the user sends a registration request. In steps, the S-CSCF will send a request to the (MAR) HSS to download the user profile and save the

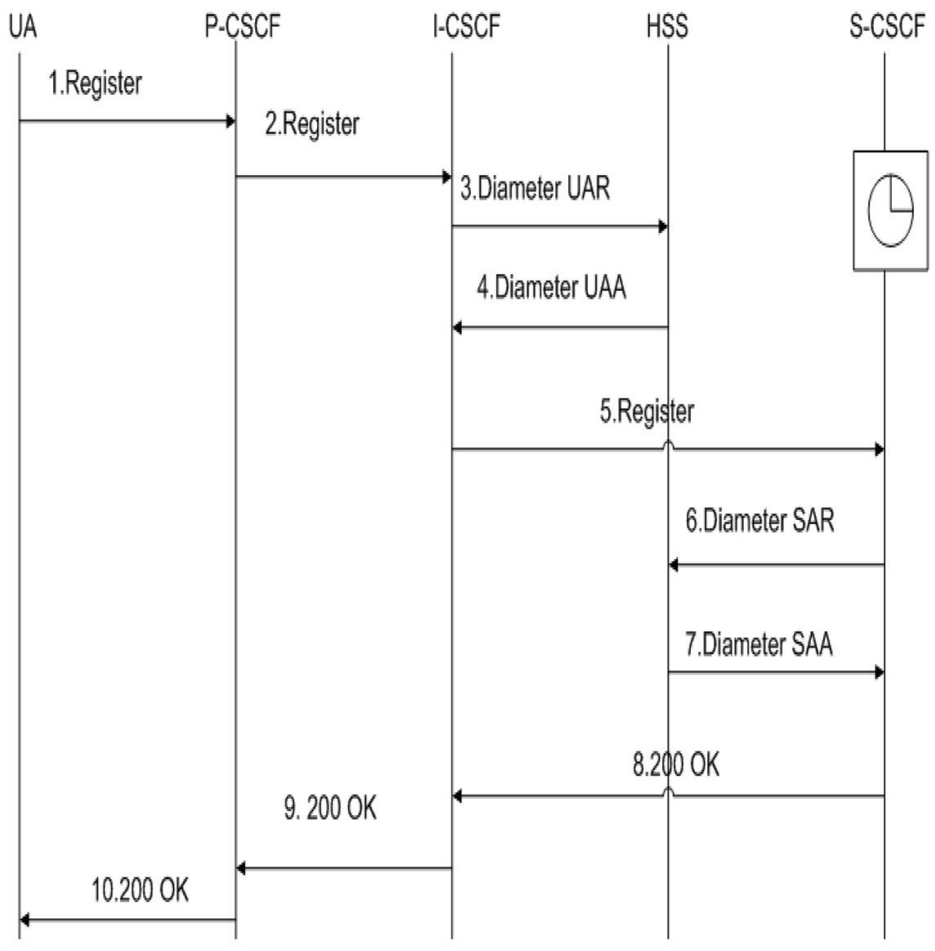

Figure 3 Cached registration scheme 
S-CSCF address in the HSS [7]. After this the S-CSCF will send an unauthorized message to the user. The message includes a challenge to the user the challenge is part of IMS security features. In steps, the S-CSCF will send a SAR message to the HSS. If the authentication is successful, the S- CSCF will inform the HSS that the user is now registered and request to download the profile (see Appendix A).

The proposed caching scheme in this work suggests adding a timer to the $\mathrm{S}-\mathrm{CSCF}$, the timer is set to represent the time of the handover delay associated with the area of coverage of IMS. The S-CSCF will start the timer. While the timer is decreasing the S-CSCF will keep the user profile and authentication. When the timer expires the S-CSCF deletes the user profile and authentication. In this case, if a registration request is received a full registration scheme needs to be performed. Caching the user data during the life of the timer will cut down on the amount of registration signaling and therefore reduces the delay time. Considering the user authentication information is kept within the S$\mathrm{CSCF}$ before the timer expires, the procedure suggests eliminating the security challenge proposed to the user. This procedure is suggested when the IMS coverage area has high traffic of registration requests arriving to the IMS system. In addition, it can be implemented in case of emergency, or spike change in user population in the area which affects the registration requests rate.

In order to capture and show the effects of different mobility models on the system, this work presents in what follows four case studies for a given area with a different scenario of mobility model in each case. The intention of this presentation is to bring to light the degree with which the IMS system is affected by the user's mobility pattern. Several realistic mobility models are utilized to represent users' mobility patterns in a coverage area. This work focuses on exploring mobility models that have not been considered before for delay effects on IMS. Users need to register every time a handover occurs in the system. Users with high mobility have higher registration rate. These requests increase the overall delay in the system and consequently degrade QoS. For this work the mobility models were generated using Matlab simulation tool. These scenarios incorporate the expected handover rate when users move from one networks to another in a hybrid network environment. The hybrid networks represent $4 \mathrm{G}$ networks (Wimax and LTE) loosely coupled with WLAN (see Appendix B).

Random Waypoint Model: The Random Waypoint Model is the most frequent model used in the simulations. The Random waypoint model is very similar to the Random Walk Model except that Random Waypoint Model has introduced the pause time parameter to the Random Walk Model. This is done 
in order to smooth out the node movement and prevent it from having sharp turns and changes in the velocity [8].

Random Walk Model: The Random walk mobility model is a user mobility model which is related to Brownian motion originally described mathematically by Einstein. It is used in different fields to resemble objects with random movements in a given area. The directions of the users and speed are selected randomly within a given range. For every new time slot, the new direction of the moving node is chosen from $(0,2 \pi)$ and the speed usually has a Gaussian distribution from $\left[0, V_{m}\right]$. The pause time for this model is zero. This model is memoryless since the direction and speed do not depend on future or past values [8], [9].

Manhattan Grid Model: Manhattan grid can represent the movement of users in a crowded city center. In the simulations it is assumed that buildings in the city center provide users with WiFi connections. Thus users need to perform handover when they step in or out of the WiFi into the 4G network. IMS in this case will be affected with handovers requests [10].

Fluid Flow Model: Users in the fluid flow model start at one point and then move in a uniformly distributed direction over $(0,2 \pi)$. They move with an average velocity of $\mathrm{v}$ and density of $\rho$ and the area boundary length is $L$ [11], [12]. The following equation gives $R$ which represents the rate of registration.

$$
R=\frac{\rho v L}{\pi}
$$

\section{Modeling, Simulation, and Analysis}

IMS Model: IMS was modeled using the Arena ${ }^{\circledR}$ simulation tool [13]. A simulation model was built to identify and analyze the total delay experienced by users when requesting IMS services. The total delay includes the registration delay and the different services delays. To model the IMS system's proxy server, (usually located in the visitor network) the simulation model assumed 10 proxies spread over the entire coverage area. Since IMS services follow different flow diagrams, the services pose different loads on the main IMS servers. Although the registration process is not a part of the initially defined services, it was considered as such in the model since every user of the system needs to register with the IMS before requesting any service. The overall performance measure considered in this study involved the Total Delay $\left(\mathrm{D}_{\mathrm{T}}\right)$ experienced by users when requesting IMS services. The total delay was 
defined as the summation of the Transmission Delay $\left(D_{t}\right)$, Queuing Delay $\left(D_{q}\right)$ and Processing Delay $\left(D_{p}\right)$, as follows:

$$
D_{T}=D_{t}+D_{q}+D_{p}
$$

Where, the Transmission Delay was defined as the delay time which occurs when sending the packets from one end to another, the Processing Delay was defined as the time it takes for the servers to process a request or query, and the Queuing Delay was defined as the time the IMS message spends in the queue of the system. Only queuing and processing delay were considered in this work. Each of the IMS servers was modeled as a network of M/M/1 queues with feedback representing the message transfer between those servers. The processing rate had an exponential distribution with a mean of 5000. The applications layer and data base delay were not considered. All the results were obtained from 5 hour simulation periods with a warm up period of 20 minutes, each simulation was replicated 4 times. The results were within 95\% confidence intervals. The Arena model reflects the amount of system delay experienced in the model compared to the different arrival rates.

Mobility Models Simulation: The work also focus on the effects of different mobility models on the IMS system. The effects of mobility patterns appeared in the form of handovers rate between networks. The user needs to perform handover when moving from one network to another. The simulation adopted an area covered by 4G network and few areas with WiFi. All the simulations have had the same assumed parameters in order to insure unified results. The following assumptions were made: the simulation area was taken to be 400 by $400 \mathrm{~m}$; the number of users were 200; and velocity of the users varies among mobility models. The simulations were carried out for 1000 iterations. Each iteration was considered a time unit of 100. The simulation was carried out by reflecting the characteristics of the considered mobility models (Fluid Flow, Random Walk, Random Way Point, Manhattan Grid). These characteristics represent the nature of the movements, the nature of the environment, distribution of the networks and users behaviors etc. During the simulation when users move from one network to another, it will trigger a handover [14]. The handover counter increases by one. The average number of handover $\left(H_{\text {average }}\right)$ is given by

$$
H_{\text {average }}=\frac{1}{U} \sum_{k=0}^{U} H_{i}(k)
$$


where $U$ is the number of total iterations, and $k$, the $k^{t h}$ experiment. The following section describes the mobility model algorithms implemented in the simulations together with the distribution of the handover for each model.

The proposed cached registration scheme focuses on reducing the queuing delay and hence the registration by caching the users' information for a given time. It trims down the signaling among IMS servers. The user profile and authentication information are cached in the S-CSCF. When the S-CSCF receives registration request and finds the user authentication and profile are available, it will send an update to the HSS for the registration and authentication. When the user information is cached the amount of signaling is reduced as shown in Figure 3. Both P-CSCF and S-CSCF have the same number of queries which is equal to 2 . While the I-CSCF is equal to 3. Thus reduce the queuing delay associated with registration procedure [15] as shown

$$
\begin{gathered}
f W_{q}(t)=\mu(1-p) e^{-\mu(1-p) t} \\
\text { Let } \alpha=\mu(1-p) \\
f W_{q}(t)=\alpha e^{-\alpha t}
\end{gathered}
$$

Where $\mu$ is the mean service rate, $\lambda$ mean arrival rate and $p$ is the utilization. Below it shows the total waiting time distribution function for each message in a flow diagram. The total waiting time distribution function is equivalent to the convolution of a waiting time distributions for each server query. The number of registration queries to P-CSCF and S-CSCF is equal to 4 and for I-CSCF the number of queries is 6 as seen in Figure 2. The queries between the user and P-CSCF are not included. In the registration flow some servers have more queries than others and the utilization function differs from one server to another.

$$
\begin{gathered}
p_{1}=p_{p}=p_{s}=\frac{4 \lambda}{0.005} \\
p_{2}=p_{i}=\frac{6 \lambda}{0.005} \\
\alpha_{1}=\mu\left(1-p_{1}\right)
\end{gathered}
$$




$$
\alpha_{2}=\mu\left(1-p_{2}\right)
$$

To solve the convolution between the pdfs, the Laplace transform of the equation as shown in [16] is:

$$
L\left\{f_{\text {WTotal }}(s)\right\}=\left(\frac{\alpha_{1}^{8}}{\left(s+\alpha_{1}\right)^{4}}\right)\left(\frac{\alpha_{2}^{6}}{\left(s+\alpha_{2}\right)^{3}}\right)
$$

Transforming it to the time domain shows the function of the total waiting time for every queue as follows:

$$
L\left\{f_{W T o t a l}(s)\right\}=\left(\alpha_{1}^{4} \frac{t^{8}}{7 !} e^{-\alpha t}\right) *\left(\alpha_{2}^{3} \frac{t^{6}}{5 !} e^{-\alpha t}\right)
$$

$f_{W T \text { Tol }}$ represents the total waiting time. The following equation also shows the total waiting time while using the proposed technique:

$$
\begin{gathered}
p_{1}=p_{p}=p_{s}=\frac{2 \lambda}{0.005} \\
p_{2}=p_{i}=\frac{3 \lambda}{0.005} \\
\alpha_{1}=\mu\left(1-p_{1}\right) \\
\alpha_{2}=\mu\left(1-p_{2}\right) \\
L\left\{f_{\text {WTotal }}(s)\right\}=\left(\frac{\alpha_{1}^{4}}{\left(s+\alpha_{1}\right)^{4}}\right)\left(\frac{\alpha_{2}^{3}}{\left(s+\alpha_{2}\right)^{3}}\right) \\
L\left\{f_{\text {WTotal }}(s)\right\}=\left(\alpha_{1}^{4} \frac{t^{4}}{3 !} e^{-\alpha t}\right) *\left(\alpha_{2}^{3} \frac{t^{3}}{2 !} e^{-\alpha t}\right)
\end{gathered}
$$

Clearly, security of the system is an important factor and therefore must be suitably incorporated. The cached scheme may be introduced in special cases such as when the system experiences an unusual level of registration requests (example in case of emergency or sports events). The reduction in the delay increases when there is a substantial increase in the registration arrival rate that will not fit with the design of the IMS. To implement the scheme a timer needs to be added to the S-CSCF and a software modification to allow the users to register without the need to have security challenge during the time the timer has not expired. 
Heretofore, the impact of different mobility models on the application layer has not received much attention. In this work, we investigate the degree with which different mobility patterns affect the application layer, in particular, the IMS signaling and server loads. The mobility patterns considered reflect different city environments, such as highways represented by fluid flow, city centers represented by the Manhattan grid, and pedestrians represented by random walk pattern. The impact of these mobility models is analyzed in the form of the rate of the expected number of handovers per second. The results were obtained from the simulation models created. Some mobility models have very little impact in comparison with other mobility models which have considerable impact on the system. In addition the cached registration scheme model is tested on these mobility models to see their effect in each environment. The cached scheme exhibits little to no effects on some mobility model and has significant impact on others as shown in the following section.

\section{Results}

From the created IMS model, the registration scheme was modeled to see the delay encountered due to the IMS servers while performing the registration procedure. Figure 4 shows the waiting time in each IMS server encountered by the signaling messages launched by the registration procedure for different arrival rates. The cached registration scheme is then implemented in the model. In comparison with IMS registration scheme without caching, the cached scheme provided a significant reduction in the delay experienced in the system. Figure 5 shows the delay in both schemes for different arrival rates.

While the cached registration scheme has shown an improvement in terms of delay, studying its impact on IMS with different mobility patterns provides the network designers an idea of when or where to implement it. The simulation results show the handovers distribution function for all scenarios. Each scenario exhibits a normal-like distribution function with different means and variances. Figures 6, 7, 8, and 9 show the distribution functions of Random Way Point, Random Walk, Manhattan Grid and Fluid Flow, respectively. Network designers will most likely benefit from these results by recognizing the capacity of the resources that need to be put in place to cater to the demands placed on the resources by each of these mobility models.

The results show the expected registration request delay in a city with different mobility patterns. The registration request arrival rate is not the only load factor on the servers, but other types of service requests are expected to 


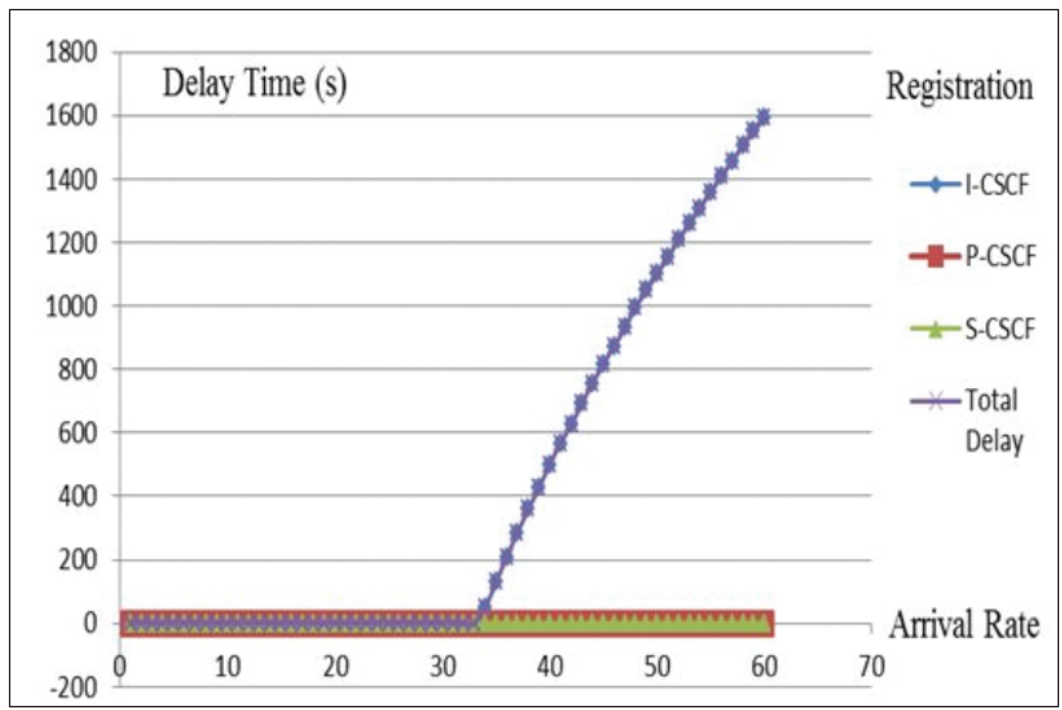

Figure 4 Registration delay (queuing and processing) in IMS servers

Table 1 Number of handovers/second for each mobility model

\begin{tabular}{lc}
\hline Mobility Model Type & Average Handovers/s \\
\hline Random Walk & 6 \\
Fluid Flow & 33 \\
Random Way Point & 3 \\
Manhattan Grid & 56 \\
\hline
\end{tabular}

load the system. Service providers should study the area mobility different patterns representing a coverage area. From the distributions the average handovers per second may be calculated, which is given in Table 1 for different mobility models.

As already mentioned, users moving from one network to another network trigger handover, and, consequently, registration requests to the IMS. The expected handover of each model was used as an input for the IMS model to test the effectiveness of the cached registration scheme with mobility patterns of the user. Table 2 shows the delay associated with each mobility model on IMS system for the generic registration scheme and the proposed cached scheme. As can be seen in Table 2, random walk model produces an average handover of 6 per second while fluid flow produces 33 handovers per second, indicating that different mobility patters impact the handover rate differently. 


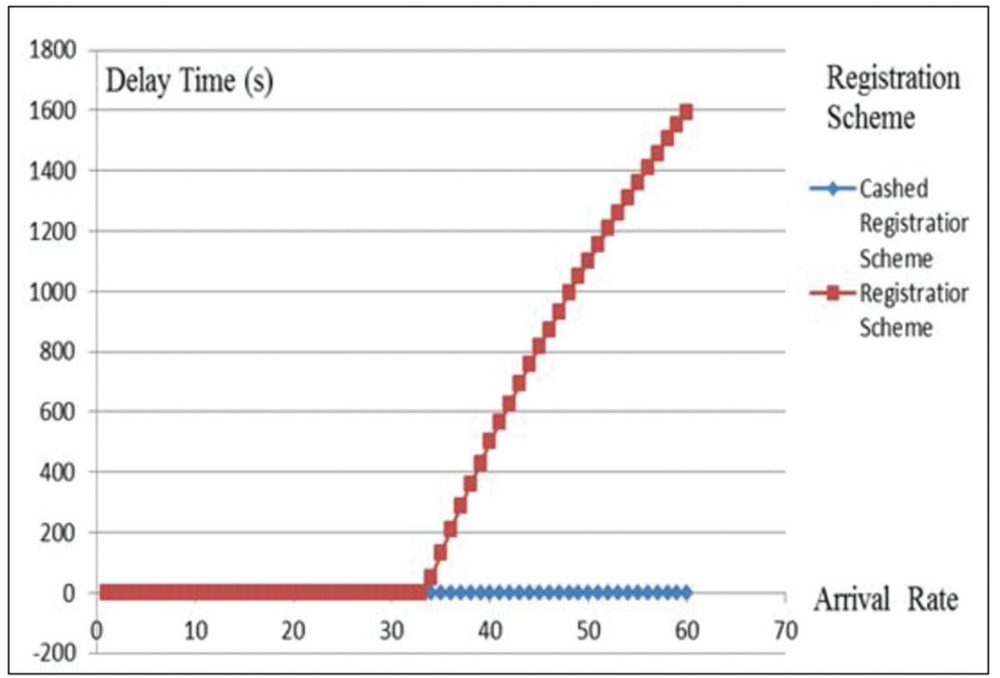

Figure 5 Registration scheme and cached registration scheme delay (queuing and processing)

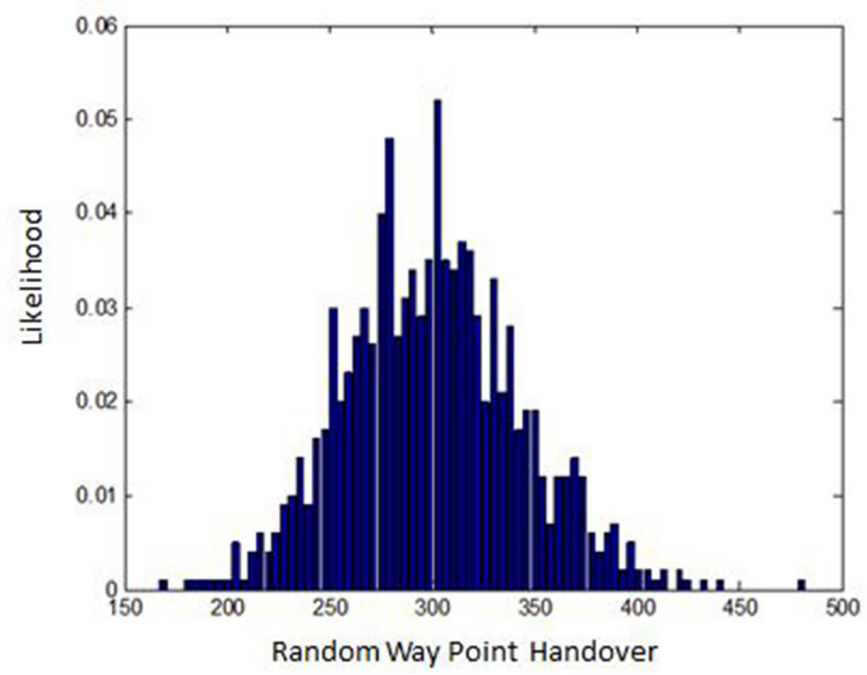

Figure 6 Random way point model handover distribution

Based on the results shown, service providers need to allocate enough resources for the registration rates in areas such as city centers and areas with a population of users with high mobility. The caching scheme can be very effective on areas with high handover rates and high mobility and may not 


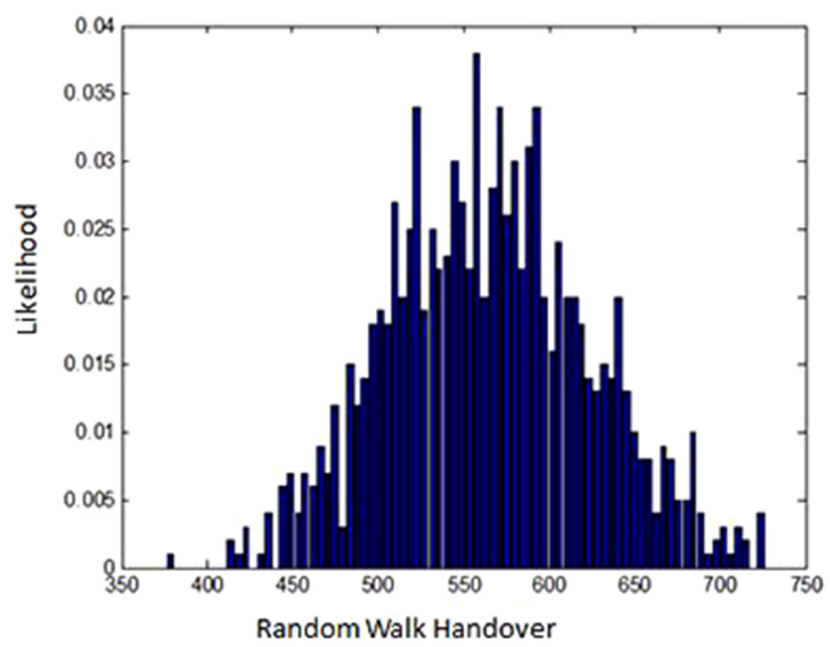

Figure 7 Random walk model handover distribution

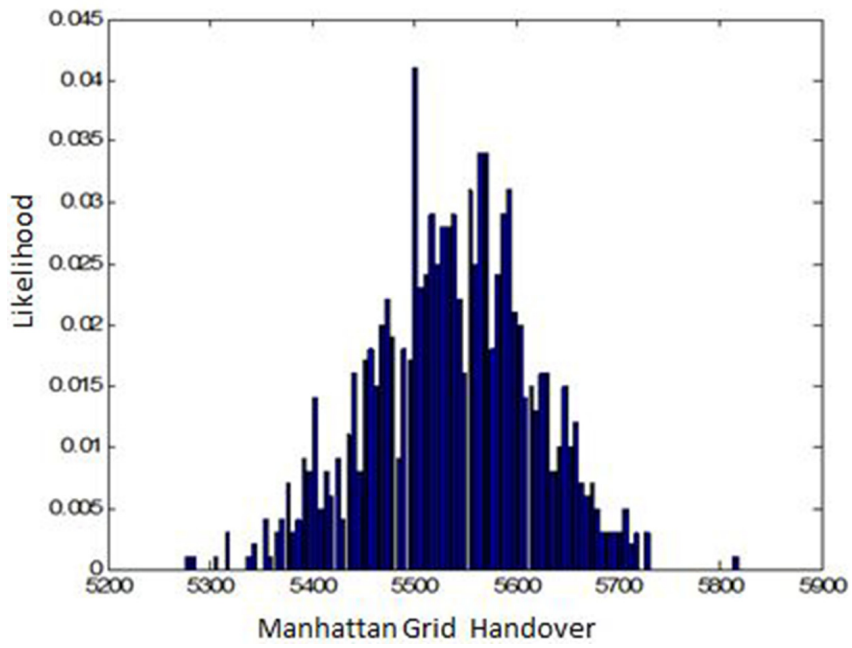

Figure 8 Manhattan grid model handover distribution

have substantial effects on users with mobility patterns similar to random walk and random way point. However, the impact of the cached scheme is more apparent in patterns similar to the fluid flow and Manhattan grid.

The mobility models considered would normally represent different aspects of a coverage area. Consequently, in most cases a single mobility model is not adequate to represent a service area. Thus, in a typical city with 


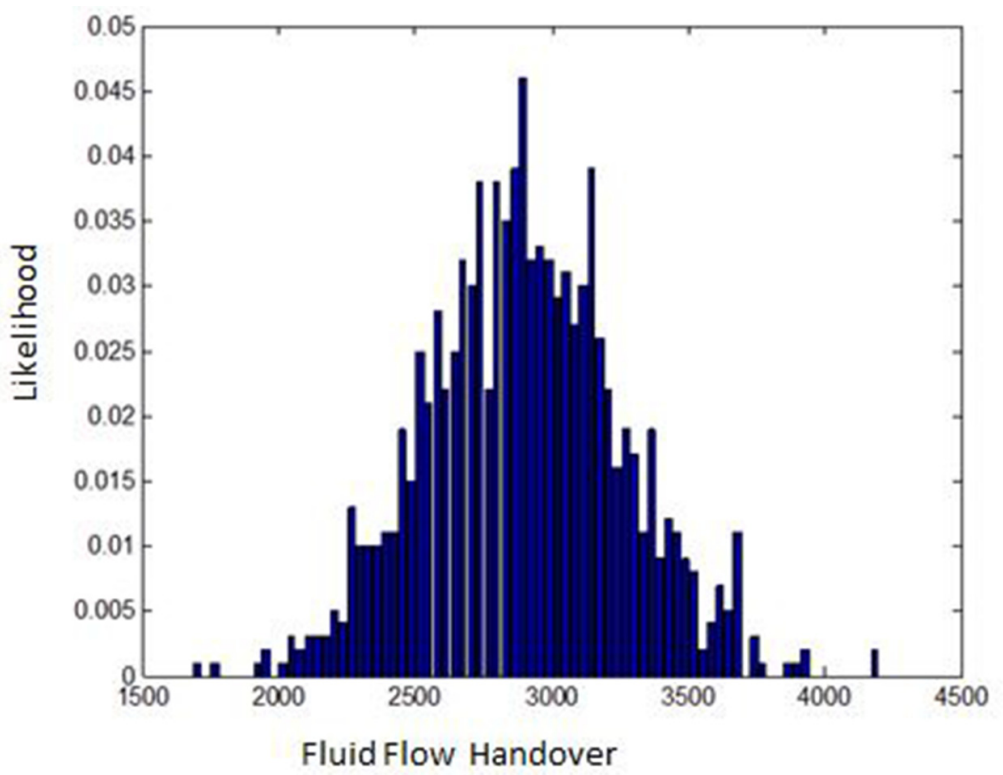

Figure 9 Fluid flow model handover distribution

Table 2 Handover delays for each mobility model for both registration scheme

\begin{tabular}{lll}
\hline $\begin{array}{l}\text { Mobility Model } \\
\text { Type }\end{array}$ & $\begin{array}{l}\text { Delay with Default } \\
\text { Registration Scheme }\end{array}$ & $\begin{array}{l}\text { Delay with Cached } \\
\text { Registration Scheme }\end{array}$ \\
\hline Random Walk & 0.0109889 & 0.000495 \\
Fluid Flow & 51.0 & 0.03 \\
Random Way Point & 0.004940 & 0.00023747 \\
Manhattan Grid & 1407.068 & 0.027 \\
\hline
\end{tabular}

highways, city center, universities and airports different types of mobility patterns should be integrated for a good representation of the IMS serservice area of that city. Averaging out the expected handover rate per second for all four mobility models and applying this average value to the IMS model. Table 3 shows the Mobility Models handover average with the delay associated with the default registration scheme and the cached registration scheme.

Table 3 Mobility models average handover and delay for each registration scheme

\begin{tabular}{llll}
\hline & $\begin{array}{l}\text { Average } \\
\text { Handover/Second }\end{array}$ & $\begin{array}{l}\text { Default Registration } \\
\text { Delay in Seconds }\end{array}$ & $\begin{array}{l}\text { Cached Registration } \\
\text { in Seconds }\end{array}$ \\
\hline $\begin{array}{l}\text { Average Mobility } \\
\text { Models Handovers }\end{array}$ & 25 & 0.02 & 0.0046724 \\
\hline
\end{tabular}




\section{Conclusion}

IP Multimedia Subsystem has shown promise to be the service infrastructure for next generation wireless networks. It caters to various services to the users, provides the environment for services development and $3^{\text {rd }}$ party integration. IMS is a complex system, and service providers face challenges in providing QoS users may require. This work has focused on the analysis and reduction of delay associated with handover for different mobility models. The work shows the diverse effects accompanied with different mobility models. It was concluded that the impact on the system was significant in the case of the Manhattan grid and fluid flow models as compared to the random walk and random way point patterns. Thus service providers need to consider the mobility factor in areas such as city centers, where higher numbers of handovers are expected in a given time. For delay reduction, the cached registration scheme was simulated to demonstrate that in cases where high handover rate is expected, the scheme reduces the waiting time significantly. However, with random walk and random way point, the scheme bears little to no effects in comparison to the default scheme. Considering that in most cases the service area might have multiple patterns given, an adaptive scheme might work best that may apply either the default scheme or the cached scheme depending on the mobility pattern encountered in the area. A formal development of such an adaptive scheme and its analysis remains a topic for further research.

\section{Appendix}

Appendix A

Pseude Code: Cached Registration Scheme.

1- User discontinues service with IMS.

2- S-CSCF keeps user information and authentication, S-CSCF timer start.

Timer $=$ Timer -1

3- Repeat until receiving registration request from same user OR

Timer $=0$.

If Timer $=0$.

Delete user information and authentication. Perform complete registration.

Else if Timer is not 0 perform cached registration. 
Appendix B

Pseude Code: Mobility Models.

1- Create $4 G$ and wifi Network areas.

2- Assign initial locations for the users, the locations are chosen randomly.

3- $\quad$ Based on the initial locations retrieve users network type.

4- For each user

\section{If the user mobility pattern is Random Way Point Mobility}

Do pause random selection between the values $\{0,2\}$

f pause is not zero then

User_pause $=$ User pause -1

Else pause is zero

For user's velocity Do random selection between $\{-10,10\}$.

New user location $(x, y)=$ Random selection $\{-10,10\}+$ current user

location $(x, y)$

Retrieve network type from users new location $(x, y)$

If change in the network type is detected from the previous location then

Handover $=$ handover +1

If the user mobility pattern is Random Walk Mobility

For user's velocity Do random selection between $\{-10,10\}$

New user location $(x, y)=$ Random selection $\{-10,10\}+$ current user location $(x, y)$

Retrieve network type from users new location $(x, y)$

If change in the network type is detected from the previous location then

Handover $=$ handover +1

If the user mobility pattern is Manhattan Grid Mobility

Do random selection of the user next step between $\{-3,3\}$.

New user location $(x, y)=$ Random selection $\{-3,3\}+$ current user location $(x, y)$

Retrieve user's network type.

If change in the network type is detected from the previous location

Handover $=$ handover +1

If the user mobility pattern is Fluid Flow Mobility

Do random selection of the user next step between.

New user location $(x, y)=$ Random selection $\{-3,3\}+$ current user location $(x, y)$ 
If New user location $(x, y)>$ location $(x, y)$ from the center of area. Then assign the new locations to the user Else repeat 5 until New user location $(x, y)>$ location $(x, y)$ from the center of the area.

If change in the network type is detected from the previous location Handover $=$ handover +1

5-Repeat 4 for the next user

\section{References}

[1] M. Ulvan, and R. Bestak "Delay Performance of Session Establishment Signaling in IP Multimedia Subsystem" $16^{\text {th }}$ International Conference on Systems, Signals and Image Processing, pp 1-5, 2009.

[2] M. Melnyk, A. Jukan, and C. Polychronopoulos "A Cross-Layer Analysis of Session Setup Delay in IP Multimedia Subsystem (IMS) With EVDO Wireless Transmissiona" IEEE transactions on Multimedia, vol 9, pp 869-881, 2007.

[3] Y.Lin, M. Tsai "Caching in I-CSCF of UMTS IP Multimedia Subsystem"IEEE Transactions on Wireless Communications, Vol 5, 2006.

[4] L. Al-Doski, A. Abbosh, R. Babiceanu, and S.Mohan. "Optimizing Resources Utilization in IP Multimedia Subsystems" IEEE International Systems Conference (IEEESysCon). 2012.

[5] G. Camarillo and M. Garcia-Martin "The 3G IP multimedia subsystem" John Wiley and Sons, 2nd ed., February 2006.

[6] E. Fogelstroem, A. Jonsson, and C. Perkins "Mobile IPv4 Regional Registration. Internet draft, draft-ietf-mip4-reg-tunnel-04" IETF, work in progress, 2007.

[7] $3^{\text {rd }}$ Generation Partnership Project (3GPP). IP Multimedia Subsystem (IMS), TS23.228, Rel10.

[8] J. Harri, F. Filali, and C. Bonnet "A Survey of Mobility Models for Ad Hoc Network Research" IEEE Communication Surveys and tutorials, volume 11, pp 19-41, 2009.

[9] W. Wang, and I. Akyildiz "Intersystem Location Update and Paging Schemes for Multitier wireless Networks" Proceedings of the $6^{\text {th }}$ annual international conference on Mobile computing and networking, pp 99-109, 2000.

[10] F. Bai and A. Helmy "A Survey of Mobility Model” Book Chapter, Kluwener Academic Publishers, USA. 
[11] L. Saleem, R Ghimire, and S. Mohan "An Analysis of IP Multimedia Subsystems for a Hybrid Network" In IEEE $4^{\text {th }}$ International Conference on Internet Multimedia Services Architecture and Applications (IMSAA), pp 1-6, 2010.

[12] S. Mohan, and R. Jain "Two User Location Strategies for Personal Communications Services" In IEEE Personal Communications, vol1, pp 42-50, 1994.

[13] D. Kelton, R. Sadowski, and D. Sturrock "Simulation with ARENA" McGraw Hill, ISBN 0-073-37628-0, 2003.

[14] L. Saleem and S. Mohan "A Complexity Analysis of IP Multimedia Subsystems (IMS)" In First International Symposium on Advanced Networks and Telecommunications Systems (ANTS), December 2007.

[15] A. Papoulis, et al "Probability Random Variables and Stochastic Processes" McGraw Hill, ISBN 0-073-66011-6, 2002.

[16] A. Amooee and A. Falahati "On Total Call Setup Waiting Time Probability Distribution in IMS Signaling Network" $3^{\text {rd }}$ International Conference on Next Generation Mobile Application and Services, pp 33-38, 2009.

[17] A. Dutta, "System Optimization for Mobility Management," PhD Dissertation, Columbia University, 2010.

\section{Biographies}

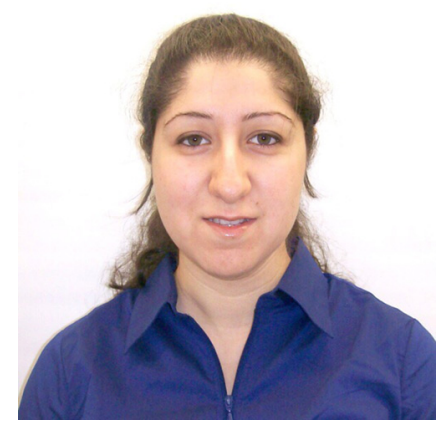

Lava Al-Doski Lava obtained both her $\mathrm{PhD}$ in Systems Engineering (Telecommunications and Networking Track) in May 2012, and Master of Science in Applied Science in December 2011, from the University of Arkansas at Little Rock. She received her Bachelor of Computer Engineering Technology in July 2005 from the Technical College of Mosul, Mosul, Iraq. She was employed as a student intern at AT\&T, Atlanta, GA, for two 
semesters during 2010/2011, where she worked on IMS and VoIP. She is currently employed at Niksun as Mobility System Engineer. She is a member of IEEE since 2008.

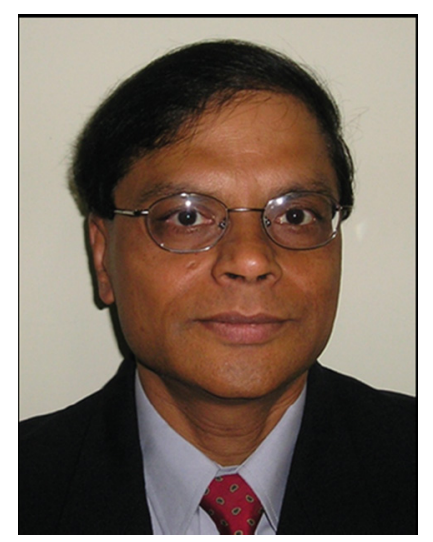

Seshadri Mohan is currently a professor in Systems Engineering Department at University of Arkansas at Little Rock, where, from August 2004 to June 2013, he served as the Chair of the Department of Systems Engineering. Prior to the current position, he served as the Chief Technology Officer with Telsima, Santa Clara, California; as Chief Technology Officer with Comverse, Wakefield, Massachusetts; as a Senior Research Scientist, with Telcordia, Morristown, NJ and as a member of the technical staff with Bell Laboratories, Holmdel, NJ. Besides his industry positions, he also served as an associate professor at Clarkson University and as an assistant professor at Wayne State University. Dr. Mohan has authored/coauthored over 100 publications in the form of books, patents, and papers in refereed journals and conference proceedings. He has co-authored the textbook Source and Channel Coding: An Algorithmic Approach. He has contributed to several books, including Mobile Communications Handbook and The Communications Handbook (both CRC Press). He holds fourteen US and international patents in the area of wireless location management and authentication strategies as well as in the area of enhanced services for wireless. He is the recipient of the SAIC Publication Prize for Information and Communications Technology. He is currently serving as the Editor-in-Chief of Advances in Network and Communications. He has served or is serving on the Editorial Boards of IEEE Personal and Nomadic Communications (now IEEE Wireless Communications), IEEE Communications Surveys and Tutorials, and IEEE Communications Magazine and has 
chaired sessions in many international conferences and workshops. He has also served as a Guest Editor for several Special issues of IEEE Network, IEEE Communications Magazine, and ACM MONET. He served as a guest editor of March 2012 IEEE Communications Magazine feature topic titled "Convergence of Applications Services in Next Generation Networks" as well as the June 2012 feature topic titled "Social Networks Meet Wireless Networks." In April 2011, he was awarded 2010 IEEE Region 5 Outstanding Engineering Educator Award. His paper titled "A Multi-Path Routing Scheme for GMPLSControlled WDM Networks," presented at the $4^{\text {th }}$ IEEE Advanced Networks and Telecommunications Systems Conference, received the best paper award. Dr. Mohan holds a Ph.D. degree in electrical and computer engineering from McMaster University, Canada, the Master's degree in electrical engineering from the Indian Institute of Technology, Kanpur, India, and the Bachelor's (Honors) degree in Electronics and Telecommunications from the University of Madras, India. 